\title{
Network Dynamics of Innovation Processes
}

\author{
Iacopo Iacopini, ${ }^{1,2}$ Staša Milojević, ${ }^{3}$ and Vito Latora ${ }^{1,4}$ \\ ${ }^{1}$ School of Mathematical Sciences, Queen Mary University of London, London E1 4NS, United Kingdom \\ ${ }^{2}$ The Alan Turing Institute, The British Library, London NW1 2DB, United Kingdom \\ ${ }^{3}$ Center for Complex Networks and Systems Research, School of Informatics and Computing, \\ Indiana University, Bloomington, Indiana 47408, USA \\ ${ }^{4}$ Dipartimento di Fisica ed Astronomia, Università di Catania and INFN, I-95123 Catania, Italy
}

(Received 19 July 2017; revised manuscript received 4 December 2017; published 24 January 2018)

\begin{abstract}
We introduce a model for the emergence of innovations, in which cognitive processes are described as random walks on the network of links among ideas or concepts, and an innovation corresponds to the first visit of a node. The transition matrix of the random walk depends on the network weights, while in turn the weight of an edge is reinforced by the passage of a walker. The presence of the network naturally accounts for the mechanism of the "adjacent possible," and the model reproduces both the rate at which novelties emerge and the correlations among them observed empirically. We show this by using synthetic networks and by studying real data sets on the growth of knowledge in different scientific disciplines. Edgereinforced random walks on complex topologies offer a new modeling framework for the dynamics of correlated novelties and are another example of coevolution of processes and networks.
\end{abstract}

DOI: 10.1103/PhysRevLett.120.048301

Creativity and innovation are the underlying forces driving the growth of our society and economy. Studying creative processes and understanding how new ideas emerge and how novelties can trigger further discoveries is therefore fundamental if we want to devise effective interventions to nurture the success and sustainable growth of our society. Recent empirical studies have investigated the emergence of novelties in a wide variety of different contexts, including science [1,2], knowledge and information [3,4], goods and products [5], language [6], and also gastronomy [7] and cinema [8]. In particular, the authors of Refs. [9-12] have looked at different types of temporally ordered sequences of data, such as sequences of words, songs, Wikipages, and tags to study how the number $S(t)$ of novelties grows with the length of the sequence $t$. They have found that the Heaps law, i.e., a power-law behavior $S(t) \sim t^{\beta}$ originally introduced to describe the number of distinct words in a text document [13], applies to different contexts, producing different values of $\beta<1$. In parallel to the empirical analyses, various models have been proposed to reproduce the innovation dynamics in different domains, such as linguistics [14,15], social systems [16], or self-organized criticality [17]. Other approaches have modeled the emergence of innovation as an evolutionary process, such as the Schumpeterian economic dynamics proposed by Thurner et al. [18] and the evolutionary game among innovators and developers proposed by Armano and Javarone [19]. Urn models (UMs) are another useful framework to study innovation processes in evolutionary biology, chemistry, sociology, economy, and text analysis [20,21]. In the classic Polya urn model [22,23], a temporal sequence of discoveries can be generated by drawing balls from an urn that contains all possible inventions. Several variations have been proposed, such as the urn model with memory, to reproduce the dynamics of collaborative tagging [11], or the more recent model by Tria and co-workers [9,24], which adds the concept of the adjacent possible $[25,26]$ to the reinforcement mechanism of Polya's urn framework.

In this Letter, we propose to model the dynamical mechanisms leading to discoveries and innovations as an edge-reinforced random walk (ERRW) on an underlying network of relations among concepts and ideas. Random walks on complex networks [27-31] have been studied at length [32]. In the context of innovation, they have been used to build exploration models for social annotation [33], music album popularity [34], knowledge acquisition [35], human language complexity [36], and evolution in research interests [37]. A special class of random walks are those with reinforcement [38-40], which have been successfully applied to biology [41] and mobility [42,43]. In particular, the concept of edge reinforcement [44-46] was introduced in the mathematical literature by Coppersmith and Diaconis [47]. Here, we will use ERWWs to mimic how different concepts are explored moving from a concept to an adjacent one in the network, with innovations being represented, in this framework, by the first discovery of nodes. As supported by empirical observations, we expect indeed the walkers to move more frequently among already known concepts and, from time to time, to discover new nodes. For this reason, we introduce and study a model in which the network is coevolving with the dynamical process taking place over it. In our model, (i) random 
walkers move over a network with assigned topology and whose edge weights represent the strength of concept associations, and (ii) the network evolves in time through a reinforcement mechanism in which the weight of an edge is increased every time the edge is traversed by a walker, making traversed edges more likely to be traversed again. As we will show, this model is able to reproduce the statistical properties observed in real data of innovation processes, i.e., the Heaps law [13], and by tuning the amount of reinforcement it can give rise to different scaling exponents. Furthermore, correlations in the temporal sequences of visited concepts and innovations will appear as a natural consequence of the interplay between the network topology and the reinforcement mechanism that controls the exploration dynamics.

Model.-Let us consider a random walker over a weighted connected graph $G(\mathcal{V}, \mathcal{E})$, where $\mathcal{V}$ and $\mathcal{E}$ are, respectively, a set of $N=|\mathcal{V}|$ nodes and a set of $K=|\mathcal{E}|$ links. Each node of the graph represents a concept or an idea, and the presence of a link $(i, j)$ denotes the existence of a direct relation between two concepts $i$ and $j$. The values of $N$ and $K$ and the topology of the network are assumed to be fixed, while the weights of the edges can change in time according to the dynamics of the walker, which, as we will see below, is in turn influenced by the underlying network. The graph at time $t$, with $t=0,1,2, \ldots$, is fully described by the non-negative time-dependent adjacency matrix $W^{t} \equiv\left\{w_{i j}^{t}\right\}$, where the value $w_{i j}^{t}$ is different from 0 if the two concepts $i$ and $j$ are related, and quantifies the strength of the relationship at time $t$. We initialize the network assuming that at time $t=0$ all the edges have the same weight, namely, $w_{i j}^{0}=1 \forall(i, j) \in \mathcal{E}$. The dynamics of the walkers is defined as follows: at each time step $t$, a walker at node $i$ jumps to a randomly chosen neighboring node $j$ with a probability proportional to the weight of the connecting edge. Formally, the probability of going from node $i$ to node $j$ at time $t$ is

$$
\operatorname{Prob}^{t}(i \rightarrow j)=\pi_{j i}^{t}=\frac{w_{i j}^{t}}{\sum_{l} w_{i l}^{t}},
$$

where the time-dependent transition probability matrix $\Pi^{t} \equiv$ $\left\{\pi_{i j}^{t}\right\}$ depends on the weights of all links at time $t$ [48]. The transition probabilities satisfy the normalization $\sum_{j} \pi_{j i}^{t}=$ $1 \forall i, t$, and we assume that $G$ has no self-loops, so that the walker changes position at each time step. On the other hand, the network coevolves with the random walk process, since every time a walker traverses a link, it increases its weight by a quantity $\delta w>0$, as illustrated in Fig. 1. This mechanism mimics the fact that the relation between two concepts is reinforced every time the two concepts are associated by a cognitive process. Formally, the dynamics of the network is the following. Every time an edge $(i, j) \in \mathcal{E}$ is traversed at time $t$, the associated weight is reinforced as

$$
w_{i j}^{t+1}=w_{i j}^{t}+\delta w .
$$

The quantity $\delta w$, called reinforcement, is the only tunable parameter of the model. The idea of a walker preferentially

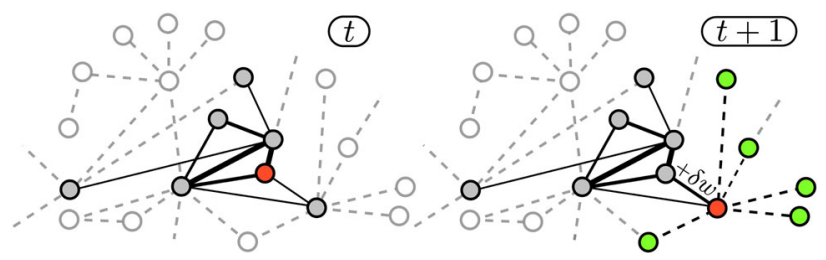

FIG. 1. Edge-reinforced random walks (ERRWs) produce a coevolution of the network with the dynamics of the walkers. At time $t$, the walker is on the red node and has already visited the gray nodes, while the shaded nodes are still unexplored. The widths of edges are proportional to their weights. At time $t+1$, the walker has moved to a neighbor (red) with probability as in Eq. (1), and the weight of the used edge has been reinforced by $\delta w$. At this point, the walker will preferentially go back, although it can also access the set of "adjacent possible" (green).

returning on its steps is in line with the classical rich-get-richer paradigm, which has been extensively used in the network literature to grow scale-free graphs [49], and is here implemented in terms of reinforcement on the edges, instead of using a random walk biased on some properties of the nodes $[38,50,51]$.

The coevolution of network and walker motion induces a long-term memory in the trajectories which reproduces, as we will show below, the empirically observed correlations in the dynamics of innovations [9]. In fact, if $i_{t}$ is a realization of the random variable $X_{t}$ denoting the position of the walker at time $t$, the conditional probability $\operatorname{Prob}\left[X_{t+1}=\right.$ $\left.i \mid i_{0}, i_{1}, \ldots, i_{t}\right]$ that, at time step $t+1$, the walker is at node $i$, after a trajectory $\mathcal{S}=\left(i_{0}, i_{1}, i_{2}, \ldots, i_{t}\right)$, depends on the whole history of the visited nodes, namely on the frequency but also on the precise order in which they have been visited [43]. The strongly non-Markovian [52] nature of the random walks comes indeed from the fact that the transition matrix $\Pi^{t}$ coevolves with the rearrangement of the weights. This makes our approach fundamentally different from the other models based on Polya-like processes. For instance, in the Tria et al. urn model [9], where an innovation corresponds to the extraction of a ball of a new color, the probability of extracting a given color (colors correspond to node labels in our model) at time $t+1$ only depends on the number of times each color has been extracted up to time $t$, and not on the precise sequence of colors. Moreover, the use of an underlying network (see Fig. 1) is a natural way to include the concept of the adjacent possible in our model, without the need of a triggering mechanism and further parameters, which are instead necessary in the UM (balls of new colors added into the urn whenever a color is drawn out for the first time) and in its mapping in terms of growing graphs considered in Supplementary Information of Refs. $[9,10]$.

Results. - We first test our model on synthetic networks, and then consider a real case where the underlying network of relations among concepts can be directly accessed and used. As a first experiment, based on the idea that concepts are organized in dense clusters connected by few longrange links, we model the relations among concepts as a 

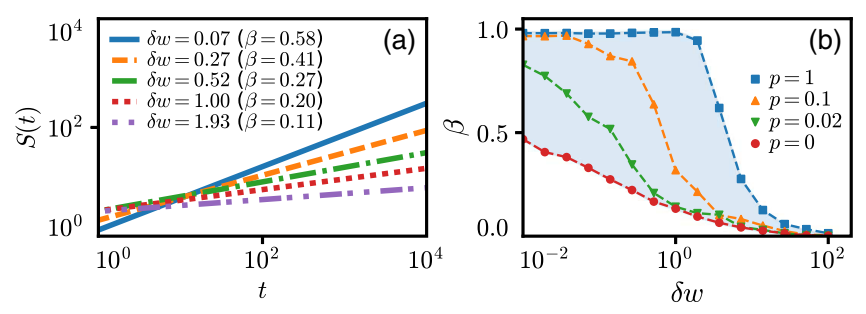

FIG. 2. ERRW on SW networks with $N=10^{5}$ and $m=1$. (a) Heaps's law and associated exponents $\beta$ obtained for different values of reinforcement $\delta w$ on a network with $p=0.02$. (b) Exponent $\beta$ as a function of the reinforcement $\delta w$ for networks with different rewiring probabilities $p$.

small-world network (SW) [53]. Our choice is supported by recent results on small-world properties of word associations [54], language networks [55], and semantic networks of creative people [56]. To construct SW networks we use the procedure proposed in Ref. [57]. Namely, we start with a ring of $N$ nodes, each connected to its $2 m$ nearest neighbors, and then we add, with a tunable probability $p$, a new random edge for each of the edges of the ring. The first thing we want to investigate is the Heaps law for the rate at which novelties happen $[9,13]$. We therefore looked at how the number of distinct nodes $S(t)$ in a sequence $\mathcal{S}$ generated by a walker grows as a function of length of the sequence $t$. Figure 2(a) shows the curves $S(t)$ obtained by averaging over different realizations of a ERRW process with reinforcement $\delta w$ on a SW network with rewiring probability $p=0.02$. All the curves can be well fitted by a power law $S(t) \sim t^{\beta}$, with an exponent $\beta$ which decreases when the reinforcement $\delta w$ increases. Finding the average number of distinct sites visited by a random walker is a well-known problem in the case of graphs without reinforcement. In particular, it has been proven that, in the absence of reinforcement, the average number of distinct sites $S(t)$ visited in $t$ steps scales as $S^{\text {ring }}(t) \sim$ $(8 t / \pi)^{1 / 2}$ [58] in one-dimensional lattices and as $S^{\mathrm{ER}}(t) \sim t$ [59] in Erdős-Rényi random graphs [60]. The transition between these two regimes has been investigated in Refs. [61-63] for SW networks with different values of $p$. Figure 2(b) reports the fitted values of the exponent $\beta$ obtained in the case of ERRW with different strength of reinforcement. The four curves refer to SW networks with rewiring probabilities $p=0,0.02,0.1$, and 1 . Notice that the previously known results, $\beta^{\text {ring }}=1 / 2$ and $\beta^{\mathrm{ER}}=1$, are recovered as limits of the two curves relative to $p=0$ and $p=1$ when $\delta w \rightarrow 0$. Furthermore, for values of $p$ in the small-world regime [64], it is possible to get values of $\beta$ spanning the entire range $[0,1]$ by tuning the amount of reinforcement $\delta w$. This means that the reinforcement mechanism we propose is able to reproduce all the Heaps exponents empirically observed [9].

Cognitive growth of science.-To show how the model works in a real case, we have extracted the empirical curves

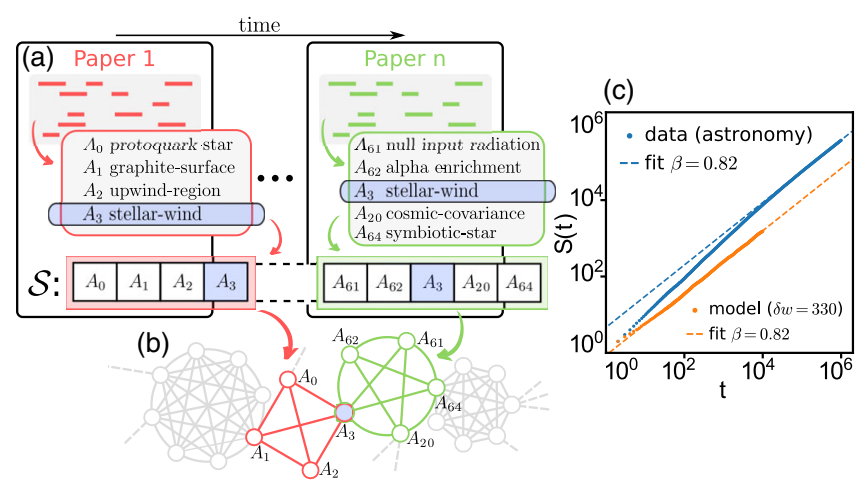

FIG. 3. Growth of knowledge in science. (a) For each scientific field, an empirical sequence of scientific concepts $\mathcal{S}$ is extracted from the abstracts of the temporally ordered sequence of papers. (b) The network of relations among concepts is constructed by linking two concepts if they appear in the same abstract. The network is then used as the underlying structure for the ERRW model. (c) The model is tuned to the empirical data by choosing the value of the reinforcement $\delta w$ that reproduces the Heaps exponent $\beta$ associated to $\mathcal{S}$.

$S(t)$ associated with a discovery process on an underlying network whose topology can be directly accessed. Specifically, we studied the growth of knowledge in modern science by analyzing 20 years (1991-2010) of scientific articles in four different disciplines, namely, astronomy, ecology, economy, and mathematics. Articles were taken from core journals in these four fields, and bibliographic records were downloaded from the Web of Science database (details in Ref. [65]). From a text analysis of each abstract, we have extracted relevant concepts as multiword phrases [66] and constructed, as in Fig. 3(a), the real temporal sequence $\mathcal{S}$ in each field from the publication date of the papers. Figure 3(c) shows that the number $S(t)$ of novel concepts in astronomy grows with the length $t$ of $\mathcal{S}$ as a power law with a fitted exponent $\beta=0.82$. Together with the real exploration sequences, we have also extracted, as illustrated in Fig. 3(b), the underlying networks of relations among concepts [67] from their co-occurrences in the abstracts, so that we do not need to rely on synthetic small-world topologies, or on the graph version of the UM (see Supplemental Material of Refs. $[9,10])$. Table I reports basic properties, such as number of nodes $N$, average node degree $\langle k\rangle$, characteristic path length $L$, and clustering coefficient $C$, for the largest components of the four networks we have constructed. Notice that different disciplines exhibit values of $\langle k\rangle$ ranging from 19 for mathematics to 172 for astronomy, but all of them have high values of $C$ and low $L$. We have then run the ERRW on each of the four networks, tuning the strength of the reinforcement $\delta w$, the only parameter of the model, so that the obtained curves for the growth of the number of distinct nodes visited by the walkers reproduce the empirical values of the exponent $\beta$. Figure 3(c) shows that, for the case of astronomy, the curve $S(t)$ of our model with $\delta w=330$ has a power-law growth with exponent $\beta=0.82$, equal to the one extracted 
TABLE I. Statistics of the network of concepts in four research fields, together with the empirical Heaps exponent $\beta$ and the value of $\delta w$ that reproduces it.

\begin{tabular}{lrrccccr}
\hline \hline Research field & Papers & $N$ & $\langle k\rangle$ & $C$ & $L$ & $\beta$ & $\delta w$ \\
\hline Astronomy & 97,255 & 103,069 & 172 & 0.41 & 2.48 & 0.82 & 330 \\
Ecology & 18,272 & 289,061 & 52 & 0.89 & 2.98 & 0.85 & 105 \\
Economy & 7,100 & 60,327 & 20 & 0.91 & 3.69 & 0.91 & 6 \\
Mathematics & 7,874 & 48,593 & 19 & 0.89 & 3.69 & 0.87 & 20 \\
\hline \hline
\end{tabular}

from the real sequence of concepts. The values of reinforcement obtained for the other scientific disciplines are reported in Table I. Notice that stronger reinforcement is required to get the same $\beta$ in networks with higher values of $\langle k\rangle$ (see Supplemental Material [68]).

Correlations. - In addition to the Heaps law, our model naturally captures also the correlations among novelties, which are a hallmark of the real exploration sequences $[9,10]$. The results for sequences generated by the ERRW model on SW networks with $p=0.02$ and $\delta w=0.01$ are plotted in Fig. 4 (different values of $p$ and $\delta w$ in Supplemental Material [68]). In particular, Fig. 4(a) shows that the frequency distribution $f(\Delta t)$ of interevent times $\Delta t$ between pairs of consecutive occurrences of the same concept is a power law, like the ones found for novelties in Wikipedia and in other data sets in Refs. [9,10]. Furthermore, the shape of $f(\Delta t)$ in our model significantly differs from that obtained by reshuffling the sequences locally and globally (see Supplemental Material [68]). Notice that $f(\Delta t)$ is the distribution of first return times, and it remains an interesting research question to investigate how first return times are linked to first passage times in the case of correlated random walks.
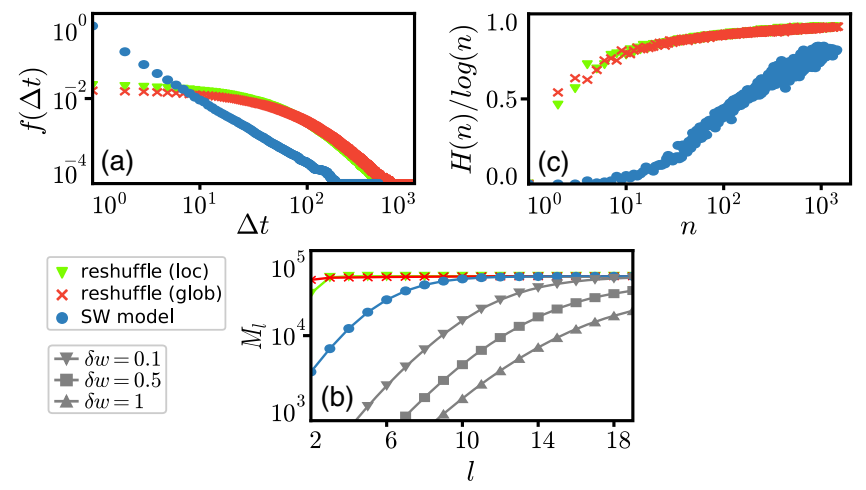

FIG. 4. Correlations among concepts produced by an ERRW $(\delta w=0.01)$ on a SW network $(p=0.02)$. (a) Frequency distribution of interevent times $\Delta t$ between consecutive occurrences of the same concept (node in our model). (b) Number $M_{l}$ of different subsequences of length $l$ as a function of $l$. (c) Normalized entropy of the sequence of visited nodes as a function of $n$, the number of times the nodes have been visited. In each panel, blue circles show average values over 20 different realizations, while triangles and crosses refer to those of (globally and locally) reshuffled sequences.
We have also looked at how $M_{l}$, the number of distinct subsequences of $\mathcal{S}$ of length $l$, grows with $l$ [69]. In Fig. 4(b) the curve $M_{l}$ generated by the ERRW model with $\delta w=0.01$ is compared to those obtained by reshuffling the sequences. The value of $M_{l}$ grows with $l$, until it reaches a plateau (equal to $T-l$, where $T=5 \times 10^{4}$ is the number of steps of the walker in the simulation) as a consequence of the finite length of $\mathcal{S}$. Interestingly, the analogous curves for the null models immediately approach the saturation value, meaning that a process without reinforcement would generate all the possible subsequences in a sequence of length $T$, while with the reinforcement this number drops down because of the correlations. In our model, the correlated sequences naturally emerge from the coevolution of network and walker dynamics, while the UM [9] requires the introduction of an additional semantic triggering mechanism to reproduce the correlations found in the data (see Supplemental Material [68] for a detailed discussion of the differences between the two models). To better characterize the correlations, we studied how homogeneously concepts occur in the sequence $\mathcal{S}$, after their first appearance. Following Tria et al. [9], we have divided the sequence $\mathcal{S}$ in $n^{(A)}$ subsequences of the same length, with $n^{(A)}$ being the total number of occurrence of $A$ in $\mathcal{S}$, and we have evaluated the Shannon entropy [70] $H^{(A)}=$ $-\sum_{s=1}^{n^{(A)}} p_{s}^{(A)} \log p_{s}^{(A)}$ for every concept $A$, where $p_{s}^{(A)}=$ $n_{s}^{(A)} / n^{(A)}$ denotes the probability of finding concept $A$ in subsequence $s$. Figure 4(c) shows the normalized average entropy $H(n)$ of concepts appearing $n$ times. Again, the large differences with respect to the null models reveal the correlated dynamics of our model. Similar results are obtained for the network of relationships among scientific concepts [68], confirming the validity of the choice of SW networks as underlying structures.

In summary, the mechanism of coevolution of network and random walks introduced in this work naturally reproduces all the properties observed in real innovation processes, including the correlated nature of exploration trajectories. With the topology of the network being a key ingredient of the model, we hope our framework will be found useful in all cases where the network can be directly reconstructed from data, as in the study of scientific innovations reported here.

We acknowledge support from EPSRC Grant No. EP/ N013492/1.

[1] A. Rzhetsky, J. G. Foster, I. T. Foster, and J. A. Evans, Proc. Natl. Acad. Sci. U.S.A. 112, 14569 (2015).

[2] R. Sinatra, D. Wang, P. Deville, C. Song, and A.-L. Barabási, Science 354, aaf5239 (2016).

[3] M. Andjelković, B. Tadić, M. M. Dankulov, M. Rajković, and R. Melnik, PLoS One 11, e0154655 (2016). 
[4] G. C. Rodi, V. Loreto, and F. Tria, PLoS One 12, e0170746 (2017).

[5] F. Saracco, R. Di Clemente, A. Gabrielli, and L. Pietronero, PLoS One 10, e0140420 (2015).

[6] A. Puglisi, A. Baronchelli, and V. Loreto, Proc. Natl. Acad. Sci. U.S.A. 105, 7936 (2008).

[7] T. Fink, M. Reeves, R. Palma, and R. Farr, Nat. Commun. 8, 2002 (2017).

[8] S. Sreenivasan, Sci. Rep. 3, 2758 (2013).

[9] F. Tria, V. Loreto, V. D. P. Servedio, and S. H. Strogatz, Sci. Rep. 4, 5890 (2014).

[10] B. Monechi, A. Ruiz-Serrano, F. Tria, and V. Loreto, PLoS One 12, e0179303 (2017).

[11] C. Cattuto, V. Loreto, and L. Pietronero, Proc. Natl. Acad. Sci. U.S.A. 104, 1461 (2007).

[12] C. Cattuto, A. Baldassarri, V. D. Servedio, and V. Loreto, arXiv:0704.3316.

[13] H.S. Heaps, Information Retrieval: Computational and Theoretical Aspects (Academic Press, Inc., New York, 1978).

[14] M. Gerlach and E. G. Altmann, Phys. Rev. X 3, 021006 (2013).

[15] L. Lü, Z.-K. Zhang, and T. Zhou, Sci. Rep. 3, 1082 (2013).

[16] M. M. Dankulov, R. Melnik, and B. Tadić, Sci. Rep. 5, 12197 (2015).

[17] B. Tadic, M. M. Dankulov, and R. Melnik, Phys. Rev. E 96, 032307 (2017).

[18] S. Thurner, P. Klimek, and R. Hanel, New J. Phys. 12, 075029 (2010).

[19] G. Armano and M. A. Javarone, Sci. Rep. 7, 1781 (2017).

[20] M. V. Simkin and V. P. Roychowdhury, Phys. Rep. 502, 1 (2011).

[21] L. Marengo and P. Zeppini, J. Evol. Econ. 26, 171 (2016).

[22] F. M. Hoppe, J. Math. Biol. 20, 91 (1984).

[23] G. Pólya, Annales de l'I.H.P. 1, 117 (1930).

[24] V. Loreto, V. D. Servedio, S. H. Strogatz, and F. Tria, Creativity and Universality in Language (Springer, New York, 2016), pp. 59-83.

[25] S. A. Kauffman, Investigations: The Nature of Autonomous Agents and the Worlds They Mutually Create. SFI working papers (Santa Fe Institute, 1996).

[26] P. Gravino, B. Monechi, V. Servedio, F. Tria, and V. Loreto, in Proceedings of the Seventh International Conference on Computational Creativity, 2016 (Sony CSL Paris, Paris, France, 2016).

[27] R. Albert and A.-L. Barabási, Rev. Mod. Phys. 74, 47 (2002).

[28] M. E. Newman, SIAM Rev. 45, 167 (2003).

[29] S. Boccaletti, V. Latora, Y. Moreno, M. Chavez, and D.-U. Hwang, Phys. Rep. 424, 175 (2006).

[30] A. Barrat, M. Barthelemy, and A. Vespignani, Dynamical Processes on Complex Networks (Cambridge University Press, Cambridge, England, 2008).

[31] V. Latora, V. Nicosia, and G. Russo, Complex Networks: Principles, Methods and Applications (Cambridge University Press, Cambridge, England, 2017).

[32] N. Masuda, M. A. Porter, and R. Lambiotte, arXiv: 1612.03281 [Phys. Rep. (to be published)].

[33] C. Cattuto, A. Barrat, A. Baldassarri, G. Schehr, and V. Loreto, Proc. Natl. Acad. Sci. U.S.A. 106, 10511 (2009).

[34] B. Monechi, P. Gravino, V. D. P. Servedio, F. Tria, and V. Loreto, R. Soc. Open Sci. 7, 170433 (2017).
[35] H. F. de Arruda, F. N. Silva, L. d. F. Costa, and D. R. Amancio, Information Sciences (NY) 421, 154 (2017).

[36] P. Allegrini, P. Grigolini, and L. Palatella, Chaos Solitons Fractals 20, 95 (2004).

[37] T. Jia, D. Wang, and B. K. Szymanski, Nat. Hum. Behav. 1, 0078 (2017).

[38] J. Gómez-Gardeñes and V. Latora, Phys. Rev. E 78, 065102 (2008).

[39] E. Agliari, R. Burioni, and G. Uguzzoni, New J. Phys. 14, 063027 (2012).

[40] R. Pemantle et al., Probab. Surv. 4, 1 (2007).

[41] D. Boyer and C. Solis-Salas, Phys. Rev. Lett. 112, 240601 (2014).

[42] J. Choi, J.-I. Sohn, K.-I. Goh, and I.-M. Kim, Europhys. Lett. 99, 50001 (2012).

[43] M. Szell, R. Sinatra, G. Petri, S. Thurner, and V. Latora, Sci. Rep. 2, 457 (2012).

[44] F. Merkl and S. W. Rolles, Dynamics and Stochastics: Festschrift in Honor of M.S. Keane edited by D. Denteneer, F. den Hollander, and E. Verbitskiy, Lect. Notes Monograph Ser. Vol. 48 (2006), pp. 66-77.

[45] M. S. Keane, S. W. Rolles et al., Verhandelingen KNAW 52 (2000).

[46] J. G. Foster, P. Grassberger, and M. Paczuski, New J. Phys. 11, 023009 (2009).

[47] P. Coppersmith and D. Diaconis (unpublished).

[48] T. M. Cover and J. A. Thomas, Elements of Information Theory (John Wiley \& Sons, New York, 2012).

[49] A.-L. Barabási and R. Albert, Science 286, 509 (1999).

[50] M. Bonaventura, V. Nicosia, and V. Latora, Phys. Rev. E 89, 012803 (2014).

[51] V. Sood and P. Grassberger, Phys. Rev. Lett. 99, 098701 (2007).

[52] C. W. Gardiner, Stochastic Methods (Springer-Verlag, Berlin, 1985).

[53] D. J. Watts, Small Worlds: The Dynamics of Networks between Order and Randomness (Princeton University Press, Princeton, NJ, 1999).

[54] P. Gravino, V. D. Servedio, A. Barrat, and V. Loreto, Adv. Complex Syst. 15, 1250054 (2012).

[55] A. E. Motter, A. P. S. de Moura, Y.-C. Lai, and P. Dasgupta, Phys. Rev. E 65, 065102 (2002).

[56] M. Benedek, Y. N. Kenett, K. Umdasch, D. Anaki, M. Faust, and A. C. Neubauer, Think. Reas. 23, 158 (2017).

[57] M. E. J. Newman and D. J. Watts, Phys. Rev. E 60, 7332 (1999).

[58] A. Dvoretzky and P. Erdös, in Proceedings of the 2nd Berkeley Symposium on Mathematics, Statistics, and Probability (University of California Press, Berkeley, CA, 1950), pp. 353-367.

[59] C. De Bacco, S. N. Majumdar, and P. Sollich, J. Phys. A 48, 205004 (2015).

[60] P. Erdös and A. Rényi, Publ. Math. (Debrecen) 6, 290 (1959).

[61] F. Jasch and A. Blumen, Phys. Rev. E 63, 041108 (2001).

[62] J. Lahtinen, J. Kertész, and K. Kaski, Phys. Rev. E 64, 057105 (2001).

[63] E. Almaas, R. V. Kulkarni, and D. Stroud, Phys. Rev. E 68, 056105 (2003).

[64] A. Barrat and M. Weigt, Eur. Phys. J. B 13, 547 (2000).

[65] S. Milojević, PLoS One 7, e49176 (2012). 
[66] S. Milojević, J. Inform. 9, 962 (2015).

[67] A. Baronchelli, R. Ferrer-i Cancho, R. Pastor-Satorras, N. Chater, and M. H. Christiansen, Trends Cognit. Sci. 17, 348 (2013).

[68] See Supplemental Material at http://link.aps.org/ supplemental/10.1103/PhysRevLett.120.048301 for details on the reshuffling procedure and additional simulations of ERRWs on real and synthetic networks.

[69] W. Ebeling and G. Nicolis, Chaos Solitons Fractals 2, 635 (1992).

[70] C. E. Shannon, Mob. Comput. Commun. Rev. 5, 3 (2001). 